\title{
The Wolf-Rayet + Of star binary AB7: A Warmer in the Small Magellanic Cloud+)
}

\author{
Manfred W. Pakull1) \& Luciana Bianchi2) \\ 1) Observatoire de Besançon, 25044 Besançon Cedex, France \\ 2) Osservatorio Astronomico di Turino, 10025 Pino Torinese, Italy
}

\section{Summary}

Strong nebular HeII $\lambda 4686$ recombination radiation $(\lambda 4686 / \mathrm{H} \beta=0.2)$ has recently been detected in the bright HII region N76 in the Small Magellanic Cloud by Testor \& Pakull (1989). The rather symmetric intensity distribution suggests a nebular morphology which consists of a thick outer shell comprising the $\mathrm{He}^{++}$and $\mathrm{H}^{+}$ionization fronts and a central region of warm, highly ionized gas which is surrounded by a hollow inner shell. The source of the high nebular ionization is identified with the peculiar Wolf-Rayet + Of star spectroscopic binary AB7. Optical and UV spectra (cf. Figure) show in addition to hydrogen and helium absorption lines and the NIII $\lambda 24634-41$ complex from the Of star, only broad He II and weak NVemission lines due to the Wolf-Rayet companion. The absence of other diagnostic features qualifies the evolved component as a rare, high-excitation WN star (WN2 or WN1). Subtracting from the optical spectrum of AB7 suitably scaled spectra of SMC O stars up to the point that the absorption lines disappeared, suggests that the WN 1 component contributes about $30 \%$ to the total optical light.
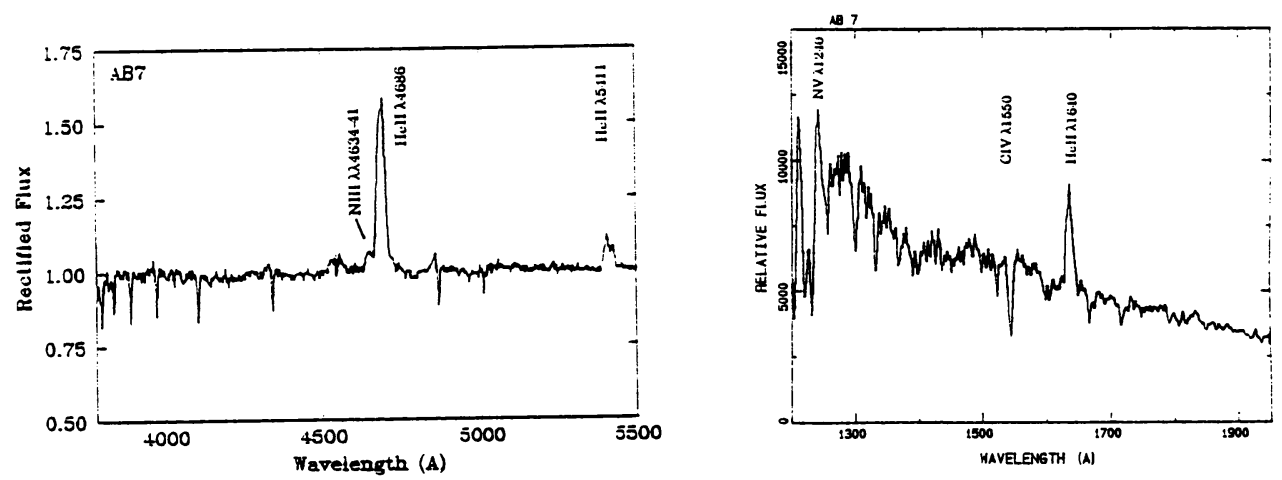

Optical and IUE spectra of the O6 IIIf + WN1 binary AB 7

From the nebular $\lambda 4686$ flux and the optical brightness of the WN1 star a black body Zanstra temperature of about $80000 \mathrm{~K}$ and a luminosity of $10^{6} \mathrm{~L}_{0}$ are derived. This result supports evolutionary models which place early WN stars near the helium main sequence of the HR diagram and provides support for the hypothesis of "Warmers", i.e. very hot luminous stars that might be responsible for the high-ionization spectra of certain active galaxies.

Although N76 appears to be predominantly ionization bound we note some leakage of ionizing photons into the surrounding IS medium which might well excite the puzzling $\lambda 4686$ halo around the nearby oxygen rich SNR 1E0102.2 -7218. 